\title{
SPECTRAL PROPERTIES OF THIOFLAVIN T AND ITS COMPLEXES WITH AMYLOID FIBRILS
}

\author{
E. S. Voropai ${ }^{a *}$ M. P. Samtsov, ${ }^{a}$ K. N. Kaplevskii, ${ }^{a}$ A. A. Maskevich, ${ }^{b}$ \\ V. I. Stepuro, ${ }^{b}$ O. I. Povarova, ${ }^{c}$ I. M. Kuznetsova, ${ }^{c}$ K. K. Turoverov, ${ }^{c}$ \\ A. L. Fink, ${ }^{\mathrm{d}}$ and V. N. Uverskii ${ }^{\mathrm{d}, \mathrm{e}}$
}

UDC 535.37

Comparative analysis of the absorption and fluorescence spectra and fluorescence excitation spectra of thioflavin T (ThT) in various solvents and in the composition of amyloid fibrils has shown that ThT, when excited in the region of the long-wavelength absorption band, fluoresces in the spectral region with a maximum at 478-484 $\mathrm{nm}$. The appearance in aqueous and alcohol solutions of a fluorescence band with a maximum near $440 \mathrm{~nm}$ has been attributed to the presence in the composition of the ThT preparations of an impurity with an absorption band in the 340-350-nm range. The literature data showing that in glycerol ThT has a wide fluorescence spectrum with two maxima are due to the artifact connected with the use of a high concentration of the dye. It has been suggested that the cause of the low quantum yield of ThT aqueous and alcohol solutions is the breakage of the system of conjugated bonds due to the reorientation of the benzothiozole and benzaminic rings of ThT in the excited state with respect to one another. The main factor determining the high quantum yield of fluorescence of ThT incorporated in fibrils is the steric restriction of the rotation of the rings about one another under these conditions. The suggestions made have been verified by the quantumchemical calculation of the ThT molecule geometry in the ground and excited states.

Keywords: thioflavin, spectrum, absorption, fluorescence, amyloid fibrils.

Introduction. The revealing and characterization of the properties of intermediate partially folded and improperly folded states arising in the process of folding-unfolding of proteins is the basic approach used in investigations to elucidate the mechanisms of folding of the polypeptide chain into a unique structure that enables the protein to perform its biological function. Stabilization of the structure of improperly folded states arising in the process of foldingunfolding of proteins is often attained due to their association and aggregation. In so doing, both disordered amorphous aggregates and ordered structures — amyloid fibrils — can arise [1]. The appearance of amorphous aggregates and their accumulation in inclusion bodies in a superexpression of recombinant proteins presents a difficult problem for biotechnology [1-6]. An ordered association of proteins with the formation of amyloid fibrils and their accumulation in different tissues and organs lead to a number of serious diseases, such as neurodegenerative Alzheimer's and Parkinson's diseases, cataracts, malignant myeloma, etc. [7-13]. Therefore, the investigation of the properties of intermediate and improperly folded states arising in the process of folding-unfolding of proteins is not only of great importance for basic research, but also of great practical importance for biotechnology and medicine. In studying the processes of folding-unfolding of proteins and the properties of the thus-arising partially folded and improperly folded states and their aggregated forms, fluorescent methods based on the recording of the natural fluorescence of proteins (see, e.g., [14-18]), the fluorescence of the hydrophobic probe of 1-anilinonaphthalene-8-sulfonate (ANS), complexing with which

*To whom correspondence should be addressed.

${ }^{\mathrm{a} B e l a r u s i a n}$ State University, 4 F. Skorina Ave., Minsk, 220050, Belarus; email: voropay@bsu.by; ${ }^{\mathrm{b}} \mathrm{Yanka}$ Kupala Grodno State University, Grodno, Belarus; ${ }^{\mathrm{C}}$ Institute of Cytology, Russian Academy of Sciences, St. Petersburg, Russia; ${ }^{\mathrm{d}}$ University of California, Santa Cruz, USA; ${ }^{\mathrm{e} I n s t i t u t e}$ of Biological Instrumentation, Russian Academy of Sciences, Pushchino, Russia. Translated from Zhurnal Prikladnoi Spektroskopii, Vol. 70, No. 6, pp. 767-773, NovemberDecember, 2003. Original article submitted December 31, 2002. 


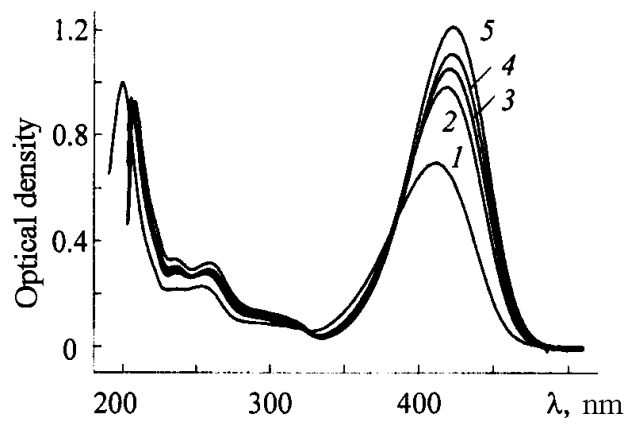

Fig. 1. Absorption spectra of ThT in water-glycerol mixtures at a glycerol content of 0 (1), 50 (2), 60 (3), 88 (4), and 97\% (5); $C_{\mathrm{ThT}}=$ const.

is a characteristic property of partially folded intermediate states of the type of a melted globule and amorphous aggregates [19], and thioflavin T (ThT) forming an intensively fluorescing complex with amyloid fibrils [20-29] are widely used. In so doing, ThT does not interact with proteins in the native, completely unfolded and partially folded denaturated states of the type of a melted globule, as well as with amorphous aggregates of proteins, and specifically interacts only with proteins in the state of amyloid fibrils. The fluorescent properties of ANS have been well studied $[30,31]$. At the same time, the mechanism of the ThT interaction with amyloid fibrils and the causes of the considerable increase in the quantum yield of fluorescence of ThT upon its incorporation into fibrils remain to be elucidated. Owing to its unique fluorescent properties, ThT is a perfect means for diagnosing the appearance of amyloid fibrils in various tissues and organs. Moreover, it is the testing of amyloid fibrils with the use of the ThT fluorescence that has become the express condition in its in vitro investigation (see, e.g., [32-35]). The number of publications along these lines is steadily increasing.

The task was to study the spectral properties of ThT in solutions with different dielectric characteristics and viscosity and in the composition of amyloid fibrils. The aim of the investigations is to elucidate the mechanism of the considerable increase (by a few orders of magnitude) in the ThT fluorescence intensity upon its incorporation into amyloid fibrils.

Materials and Methods. We used the preparation ThT (Fluka, Switzerland), ethanol, methanol, dioxane, acetone, hexane, chloroform, glycerol (Merck, Germany), cyclohexane (Fisher, USA), and 1-butanol (Baker, USA). Thioflavin was recrystallized from an acetonitrile + ethanol mixture in a 3:1 ratio. The crystals were washed on a filter with ethanol.

The absorption spectra were measured on Hitachi EPS-3T-type (Japan) and Solar PV 1251A (Belarus) spectrophotometers. Fluorescent measurements were made using a Spex FluoroMax-2 spectrofluorimeter (USA) and the spectrofluorometric laboratory facility presented in [36].

Results. ThT absorption spectra in various solvents. The intensity and the maximum position of the longwavelength absorption band of ThT are largely determined by the solvent. This band has the shortest-wavelength maximum position in the case of water solution of ThT and the longest-wavelength one - in chloroform and glycerol. The absorption spectrum of ThT in glycerol-water mixtures with increasing content of glycerol shifts towards the longwave region with a simultaneous increase in the absorption intensity (Fig. 1). In investigating the influence of solvent on the ThT absorption spectra, it has been found that ThT practically is not dissolved in hexane and cyclohexane. The dissolved part of the ThT preparations in these solvents has absorption spectra with a maximum near 340-350 nm that are most probably due to the impurity in the ThT preparations. This is evidenced by the disappearance of this absorption band in hexane and cyclohexane upon ThT recrystallization.

ThT fluorescence in various solvents. When excited in the region of the long-wave absorption band $\left(\lambda_{\operatorname{exc}}=\right.$ $440 \mathrm{~nm}$ ), ThT fluoresces with an emission-band maximum at $474 \mathrm{~nm}$. The shape and position of the fluorescence-band maximum are independent of the solvent, while the fluorescence intensity is strongly dependent on it (Fig. 2a, b). The lowest fluorescence intensity has been registered for the water solution of ThT and the highest one - for ThT in glycerol. In measuring the fluorescence excitation spectra $\left(\lambda_{\text {exc }}=480 \mathrm{~nm}\right)$, we have found that these spectra are simi- 

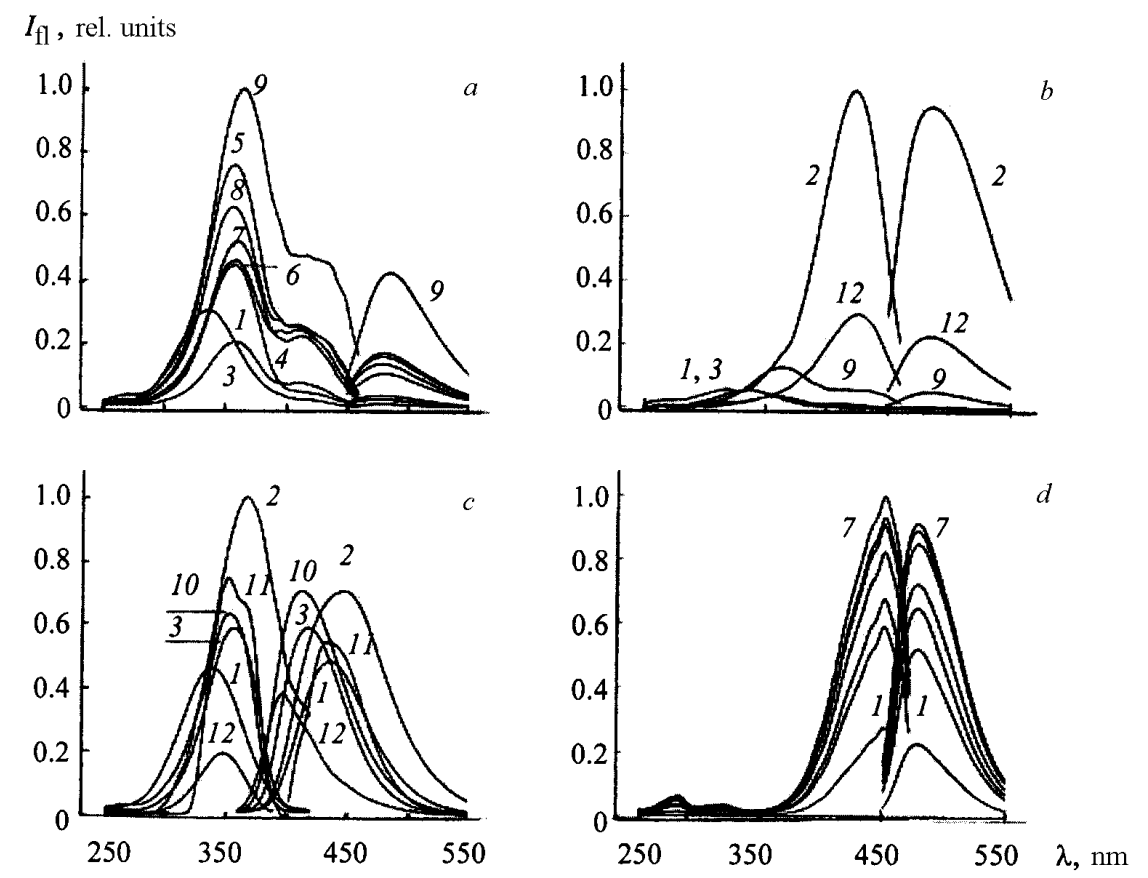

Fig. 2. Fluorescence excitation and fluorescence spectra of ThT at $\lambda_{\text {rec }}=480$ $\mathrm{nm}$ and $\lambda_{\text {exc }}=440 \mathrm{~nm}(\mathrm{a}, \mathrm{b}, \mathrm{d})$, at $\lambda_{\text {rec }}=440 \mathrm{~nm}$ and $\lambda_{\text {exc }}=340 \mathrm{~nm}(\mathrm{c}): \mathrm{a}$, b, c, in water (1), glycerol (2), ethanol (3), methanol (4), 1-propanol (5), 2propanol (6), 1-butanol (7), isopropyl alcohol (8), ethylene glycol (9), acetone (10), acetonitrile (11), and chloroform (12); $d$, in the presence of insulin fibrils of concentration from 0.02 to $0.14 \mathrm{mg} / \mathrm{ml}\left(1-7\right.$, respectively); $C_{\mathrm{ThT}}=0.05$ $\mu \mathrm{M}$.

lar in shape and maximum position to the absorption spectra only for ThT solutions in glycerol and chloroform. It was rather surprising that the ThT fluorescence spectra in water had a maximum at $340 \mathrm{~nm}$ and in alcohols - at 355-360 $\mathrm{nm}$ (Fig. 2b), i.e., in the spectral region corresponding to the minimum in the ThT absorption spectrum. Only the long-wave shoulders of the ThT excitation spectra in these solvents lie in the spectral region corresponding to the long-wave absorption band of ThT. Upon excitation in the region of the maximum of the excitation spectra $\left(\lambda_{\text {exc }}=\right.$ $340 \mathrm{~nm}$ ) for ThT in these solvents fluorescence bands with a maximum in the spectral region corresponding to the long-wave absorption band of the dye are observed (Fig. 2). At the same time, the intensity ratio $I_{340} / I_{420}$ in the ThT fluorescence excitation spectra at the wavelengths of 340 and $420 \mathrm{~nm}$ in water and alcohols upon recrystallization of the preparations decreased by a factor of 2 and 4, respectively.

Fluorescence of ThT intercalated into amyloid fibrils. The fluorescence spectra of ThT incorporated into amyloid fibrils are similar in shape and maximum position to the fluorescence spectra of ThT in the water solution upon excitation in the region of the long-wave band of the absorption spectrum and the fluorescence excitation spectra are similar to the ThT absorption spectra (Fig. 2c).

Upon the addition of amyloid fibrils to the ThT solution, the fluorescence intensity of the dye increases by a few orders of magnitude and grows with increasing concentration of fibrils (Fig. 2d). The fluorescence intensity of $0.01 \mu \mathrm{M}$ of ThT in the buffer solution in the presence of $0.02 \mathrm{mg} / \mathrm{ml}$ of insulin fibrils exceeds the ThT intensity in the water solution by a factor of $\sim 1000$.

Discussion. ThT fluorescence in water solutions and alcohols. According to the literature data [20, 21], the water solution of ThT has a fluorescence spectrum with a maximum at $438 \mathrm{~nm}$ and a fluorescence excitation spectrum at $350 \mathrm{~nm}$. The close values of the maximum position of the fluorescence excitation and radiation spectra are given in [22-25]. It should be noted that in all these works it has been shown that the fluorescence excitation and radiation 
$I_{\mathrm{fl}}$, rel. units

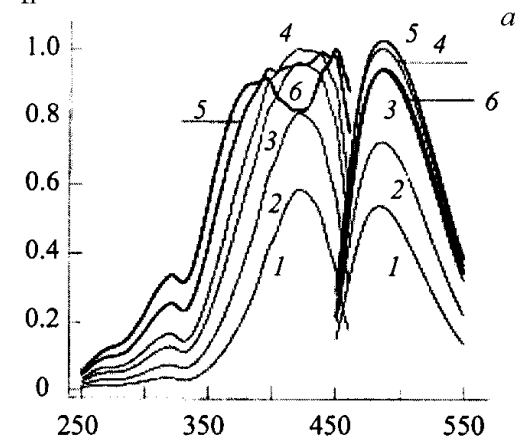

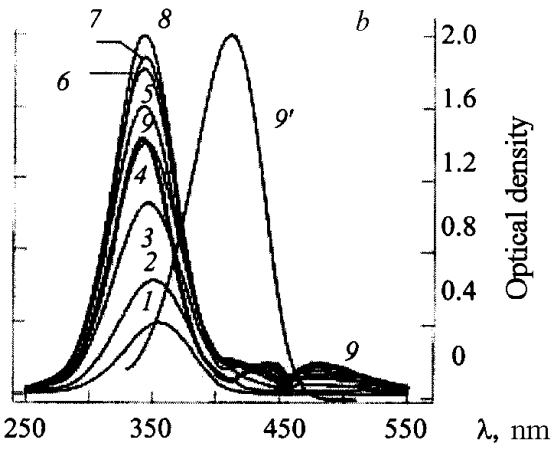

Fig. 3. Fluorescence excitation $\left(\lambda_{\text {rec }}=480 \mathrm{~nm}\right)$ and fluorescence $\left(\lambda_{\text {exc }}=440\right.$ $\mathrm{nm})$ spectra of ThT in glycerol (a) and water (b): a, $C_{\mathrm{ThT}}=5$ (1), 10 (2), 20 (3), 29 (4), 48 (5), and $65 \mu \mathrm{M}$ (6); b, 5 (1), 10 (2), 20 (3), 29 (4), 38 (5), 48 (6), 57 (7), 65 (8), and $90 \mu \mathrm{M}$ (9); 9', absorption spectrum at $C_{\mathrm{ThT}}=90 \mu \mathrm{M}$ $\left(D_{412}=2.0\right)$; solutions with $C_{\mathrm{ThT}}=5,10,20,29,38,48,57,65$, and $90 \mu \mathrm{M}$ have an optical density at the maximum of $0.01,0.08,0.25,0.48,0.65,0.81$, $1.12,1.27$, and 2.00 , respectively.

spectra are shifted towards the long-wave region upon incorporation into the fibrils. Our data point to the fact that, upon excitation in the region of the long-wave absorption band of the dye, ThT in fibrils, water, and alcohol solutions, as well as in glycerol, chloroform, and PVA film (the data are not given) fluoresces in one and the same spectral region with a maximum at 478-484 $\mathrm{nm}$ (Fig. 2a, b, d). At the same time, indeed, upon excitation in a shorter-wave region of the spectrum $\left(\lambda_{\mathrm{exc}}=340 \mathrm{~nm}\right)$ of ThT in the water solution and alcohols a fluorescence band with a maximum near $440 \mathrm{~nm}$ is observed (Fig. 2b). This band has a fluorescence excitation spectrum with maxima at $340 \mathrm{~nm}$ in water and $355-360 \mathrm{~nm}$ in alcohols.

Such an unusual spectral behavior of ThT in water and in a number of alcohols, as we see it, can only be explained if we assume the presence in the composition of the ThT preparations of an impurity having an absorption band with a maximum at 340-350 $\mathrm{nm}$ and a fluorescence with a high quantum yield. This is evidenced by the decrease in the contribution to the ThT fluorescence excitation spectrum of the band with the 340-nm maximum upon recrystallization of the preparation. It is this impurity that determines the appearance of the absorption band with a maximum near 340-350 nm upon ThT "dissolution" in hexane and cyclohexane (see above).

The appearance of the "normal" fluorescence band with a maximum at $474 \mathrm{~nm}$ upon excitation in the region of $340 \mathrm{~nm}$ can be attributed to the reabsorption of the impurity fluorescence by the ThT molecules or the nonradiative energy transfer from the impurity to the ThT molecules. It should be noted that abnormal fluorescence and fluorescence excitation spectra are only observed in the cases where the "normal" fluorescence of ThT excited in the region of the long-wave absorption band has a low quantum yield. These bands do not show up against the background of the intensive fluorescence of ThT in glycerol or chloroform.

Dependence of the spectral characteristics of ThT on its concentration in solution. Possible artifacts. The ThT excitation spectrum in glycerol obtained by us is similar in shape and maximum position of the spectrum to the absorption spectrum of the dye (Fig. 3). At the same time, according to the data of [23], the ThT excitation spectrum in glycerol represents a wide band with two maxima. From our point of view, such a character of the spectrum is likely to be due to the fact that it was registered for a solution with a high concentration of the dye $(30 \mu \mathrm{M})$. The effect is due to the character of the dependence of the fluorescence intensity on the optical density of the solution. The fluorescence intensity is proportional to the exciting-light intensity $I_{0}$, the fraction of the light absorbed by the sample $(1-T)$, and the quantum yield of fluorescence $q$ :

$$
I_{\mathrm{fl}}=k I_{0}(1-T) q,
$$


TABLE 1. Spectral-Luminescent Characteristics of ThT in Various Solvents

\begin{tabular}{|l|c|c|c|c|c|c|c||}
\hline \multicolumn{1}{|c|}{ Solvent } & $\varepsilon$ & $\begin{array}{c}\eta \cdot 10^{3}, \mathrm{~N} \cdot \mathrm{sec} / \mathrm{m}^{2} \\
\left(20^{\circ} \mathrm{C}\right)\end{array}$ & $\lambda_{\mathrm{abs}}, \mathrm{nm}$ & $\Delta \lambda_{\mathrm{abs}}, \mathrm{nm}$ & $\lambda_{\mathrm{f}}, \mathrm{nm}$ & $\Delta \lambda_{\mathrm{f}}, \mathrm{nm}$ & $\varphi_{\mathrm{f}}, \%$ \\
\hline Ethanol & 24.3 & 1.20 & 416 & 58 & - & - & 0.3 \\
\hline Methylene chloride & 8.9 & 0.43 & 432 & 51 & 504 & 75 & 1.2 \\
\hline Dichlorobenzene & 9.9 & 1.30 & 439 & 51 & 505 & 108 & 3.9 \\
\hline
\end{tabular}

where $T \equiv I / I_{0}=10^{-D}$ is the fraction of the light transmitted through the sample (transmission); $k$ is proportionality coefficient; $D$ is the optical density of the solution $(D=\varepsilon C l), l$ is the optical path, $\varepsilon$ is the molar extinction coefficient, and $C$ is the concentration. Apparently, $I_{\mathrm{fl}}=k \varepsilon l I_{0} C q$ at $D \rightarrow 0$ and $I_{\mathrm{fl}}=k I_{0} q$ at $D \rightarrow \infty$.

Thus, the fluorescence intensity is proportional to the concentration of the fluorescent substance only at low values of the optical density of the solution being investigated; at high values of the optical density (high concentration) the fluorescence intensity is independent of the concentration of the fluorescent substance in the solution. In practice, the dependence of the fluorescence intensity on the optical density of the solution does not reach a plateau, as one would expect, and upon attaining a certain optical density begins to decrease. This is due to the fact that at high optical densities the exciting light does not reach the region of the cell which the facility's detector "sees" ("internal filter" effect).

It is especially important to take into account the foregoing in measuring the excitation spectra. The optical density of the solution changes depending on the excitation spectrum. It is maximal in the region of the absorptionband maximum, which provides the possibility of a dip in the excitation spectra in working with solutions of a high optical density. Figure 3a gives the fluorescence and fluorescence excitation spectra of ThT in glycerol. At a high concentration of the dye the fluorescence intensity decreases with increasing concentration, and in the fluorescence excitation spectrum there is a characteristic dip with a minimum corresponding to the maximum of the long-wave band of the absorption spectrum. The fluorescence excitation spectrum obtained thereby is very similar in shape to the fluorescence spectrum of ThT in glycerol presented in [23].

Interestingly, for the water solution of ThT, as the dye concentration increases, at the long-wave edge of the excitation spectrum first a shoulder and at high concentrations even an additional band arises (Fig. 3b). However, these results are not associated with the formation of aggregated forms of the dye, as would seem at first glance, but are due to (for the above reasons) the appearance of a minimum in the excitation spectrum exactly corresponding to the minimum of the absorption spectrum ThT in this solvent (Fig. 3b, curve $9^{\prime}$ ).

Fluorescent properties of ThT in solvents with a different polarity and viscosity. A possible cause of the low quantum yield of ThT in the water solution and in a number of other solvents is the breakage of the system of conjugated bonds due to the reorientation of benzothiazole and benzamine rings of ThT with respect to one another. The unexcited thioflavin molecule has a near-planar configuration. Upon excitation there occurs an intramolecular charge transfer between the rings. The nonequilibrium state (with separated charges) that arises can be stabilized if the rings go out of the conjugation and one of them rotates by $90^{\circ}$. Then back charge transfer will be impossible. Transitions with emission from this state (with rings turned to one another) are assumed to have a low efficiency.

ThT molecules in the excited state can be stabilized by a polar solvent whose molecules reorientate following the rotation of the ring. This is likely to take place in the case where the solvent is water or alcohol. When the solvent is glycerol or PVA film, the microsurrounding viscosity is high and the process of reorientation of the rings does not manage to proceed within the lifetime of the excited state. In low-polarity solvents, such as dichlorobenzene and methylene chloride, the quantum yield of fluorescence of ThT is about an order of magnitude higher than in ethanol (see Table 1). Ethanol and dichlorobenzene have an approximately equal viscosity. This means that the quantum yield of fluorescence of ThT is influenced not only by the viscosity of the medium but by its polarity as well. On the other hand, the quantum yield of fluorescence of ThT in low-polarity methylene chloride is about three times lower than in low-polarity dichlorobenzene. The viscosities in these solvents also differ by a factor of $\sim 3$. Thus, the value of the quantum yield of fluorescence of ThT is determined by both the viscosity and the polarity of the solvent. Nonpolar 
solutions cannot compensate for the electrostatic interaction between the polar fragments of the thioflavin molecule and stabilize the structure of ThT with rings rotated about one another. In this case, the transition of the molecule back to the planar form and fluorescence de-excitation are possible.

The above proposals are supported by the quantum-chemical calculation of the geometry of conformers of the thioflavin $\mathrm{T}$ cation $(Z=+1)$ in the gas phase done by us. The calculation was carried out by the semiempirical PM3 method [37] with the use of the GAMESS-US quantum-mechanical package [38]. The calculations have shown that the angle between the ring planes in the ground state $\varphi=30^{\circ}$, i.e., the molecule is not strictly planar:

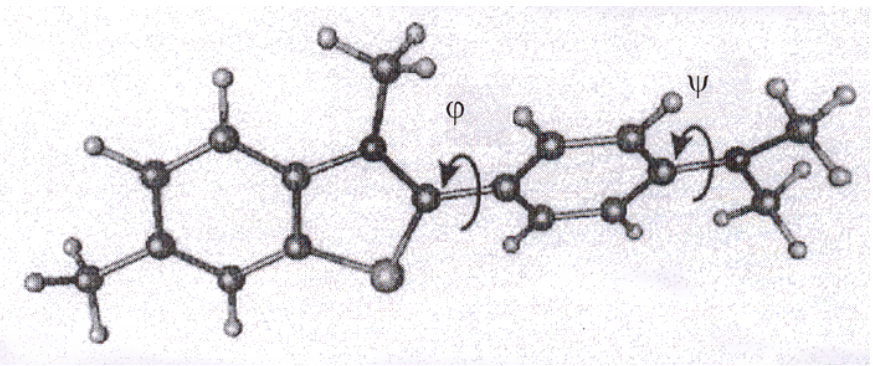

To check the possibility of rotation of the rings in the excited state, we have calculated the electronic spectrum of thioflavin by the ZINDO/S method [39], taking into account the configuration interaction. For conformations with different angles $\varphi$ obtained in optimizing the molecule geometry in the ground $S_{0}$-state, the energy of the excited $S_{1}^{*}$-state has been determined. The $S_{1}^{*}$-state energy assumed the maximum value at an angle between the rings of $\varphi=$ $90^{\circ}$, which corresponds to the above-proposed model. The barrier inhibiting the rotation of the rings about one another is small - about $2.2 \mathrm{kcal} / \mathrm{mole}$, while the potential barrier of rotational isomerization of the amino group in the benzene ring plane $\left(\varphi=0\right.$ or $\left.90^{\circ}\right)$ is $\sim 6.1 \mathrm{kcal} / \mathrm{mole}$.

Conclusion. The rigidity of the microsurrounding inhabiting the rotation of the rings about one another upon transition of the ThT molecule to the excited state is likely to be the basic factor determining the high quantum yield of fluorescence of ThT incorporated into amyloid fibrils.

This work was supported by grants from the Russian Basic Research Foundation (grant No. 02-04-81018_Bel) and the Belarusian Republic Basic Research Foundation (grant No. F02R-061), a grant from the Presidium of the Russian Academy of Sciences on physico-chemical biology, and an INTAS grant (INTAS-2001-2347).

\section{REFERENCES}

1. A. L. Fink, Fold. Des., 3, R9023 (1998).

2. C. H. Schein, Biotechnology, 7, 1141-1149 (1989).

3. S. Frankel, J. Condeelis, and L. Leinwand, J. Biol. Chem., 265, 17980-17987 (1990).

4. R. Wetzel, in: A. R. Rees, A. R. Sternberg and R. Wetzel, Protein Engineering. A Practical Approach, IRL Press, Oxford (1992), pp. 191-219.

5. R. Wetzel, Trends Biotechnol., 12, 193-198 (1994).

6. M. A. Speed, D. I. Wang, and J. King, Nat. Biotechnol., 14, 1283-1287 (1996).

7. R. W. Carrell and B. Gooptu, Curr. Opin. Struct. Biol., 8, 799-809 (1998).

8. J. W. Kelley, Structure, No. 5, 595-600 (1997).

9. J. D. Harper and P. T. Lansbury, Jr., Ann. Rev. Biochem., 66, 385-407 (1997).

10. E. H. Koo, P. T. Lansbury, Jr, and J. W. Kelly, Proc. Natl. Acad. Sci. USA, 96, 9989-9990 (1999).

11. M. Hashimoto and E. Masliah, Brain Pathol., No. 9, 707-720 (1999).

12. V. N. Uverskii (Uversky), A. Talapatra, J. R. Gillespie, and A. L. Fink, Med. Sci. Monitor, 5, 1001-1012 (1999).

13. V. N. Uverskii (Uversky), A. Talapatra, J. R. Gillespie, and A. L. Fink, Med. Sci. Monitor, 5, 1238-1254 (1999).

14. C. Bodenreider, N. Kellershohn, M. E. Goldberg, and A. Mejean, Biochemistry, 41, 14988-14999 (2002).

15. F. Hannemann, A. K. Bera, B. Fischer, M. Lisurek, K. Teuchner, and R. Bernhardt, Biochemistry, 41, 1100811016 (2002).

16. A. P. Capaldi, M. C. Shastry, C. Kleanthous, H. Roder, and S. E. Radford, Nat. Struct. Biol., 1, 68-72 (2001). 
17. K. K. Turoverov and I. M. Kuznetsova, J. Fluores., 13, 41-57 (2003).

18. I. M. Kuznetsova, O. V. Stepanenko, O. I. Povarova, A. G. Biktashev, V. V. Verkhusha, M. M. Shavlovsky, and K. K. Turoverov, Biochemistry, 41, 13127-13132 (2002).

19. G. V. Semisotnov, N. A. Rodionova, O. I. Razgulyaev, V. N. Uverskii (Uversky), A. F. Grupas', and R. I. Gilmanshin, Bioplymers, 31, 119-128 (1991).

20. H. Naiki, K. Higuchi, M. Hosokawa, and T. Takeda, Anal. Biochem., 177, $244-249$ (2989).

21. H. Haiki, K. Higuchi, K. Matsushima, A. Shimada, W. H. Chen, M. Hosokawa, and T. Takeda, Lab. Invest., 62, $768-773$ (1990).

22. H. LeVine, III, Protein Sci., 2, 404-410 (1993).

23. H. LeVine, III, Int. J. Exp. Clin. Invest., 2, 1-6 (1995).

24. H. LeVine, III, Arch. Biochem. Biophys., 342, 306-316 (1997).

25. H. LeVine, III, Methods Enzymol., 309, 274-284 (1999).

26. Y. Yoshiike, D. H. Chui, T. Akagi, N. Tanaka, and A. Takashima, J. Biol. Chem., 278, 23648-23655 (2003).

27. A. Elhaddaoui, A. Delacourte, and S. Turrell, J. Mol. Struct., 294, 115-118 (1993).

28. D. Allop, L. Swanson, S. Moore, Y. Davies, A. York, O. M. El-Agnaf, and I. Soutar, Biochem. Biophys. Res. Commun., 285, 58-63 (2001).

29. R. O. Loutfy and B. A. Arnold, J. Phys. Chem., 86, 4205-4211 (1982).

30. V. N. Uverskii (Uversky), S. Winter, and G. Lober, Biophys. Chem., 60, 79-88 (1996).

31. V. N. Uverskii (Uversky), S. Winter, and G. Lober, Biophys. Chem., 60, $79-88$ (1996).

32. J. Goers, S. E. Permyakov, E. A. Permyakov, V. N. Uverskii (Uversky), and A. L. Fink, Biochemistry, 41, 12546-12551 (2002).

33. J. R. Kumita, C. J. Waston, L. P. Choo-Smith, G. A. Wooley, and O. S. Smart, Biochemistry, 42, 4492-2298 (2003).

34. L. Zhu, X. J. Zhang, L. Y. Wang, J. M. Zhou, and S. Perett, J. Mol. Biol., 328, 235- (2003).

35. T. Ban, D. Hamada, K. Hasegawa, H. Naiki, and Y. Goto, J. Biol. Chem., 278, 16462-16465 (2003).

36. K. K. Turoverov, A. G. Biktashev, A. S. Dorofeyuk, and I. M. Kuznetsova, Tsitologiya, 40, 806-817 (1998).

37. J. J. Stewart, J. Comput. Chem., 10, 221-264 (1989).

38. M. W. Schmidt, K. K. Baldridge, and J. A. Boatz, J. Comput. Chem., 14, 1347-1363 (1993).

39. M. C. Zerner, in: K. B. Lepkowitz and D. B. Boyd (Eds.), Semiempirical Molecular Orbital Methods. Reviews in Computational Chemistry II, VCH Publishers, New York (1991), pp. 313-366. 